\title{
VLBI Observations of an Expanding Jet in Cygnus X-3
}

\author{
Lawrence A. Molnar, M. J. Reid, and J. E. Grindlay \\ Center for Astrophysics, Cambridge, MA USA
}

The X-ray binary Cyg X-3 offers a rare opportunity to study the physical conditions of the formation of a relativistic jet. While the central engines of extragalactic jets are generally poorly understood, the infrared and X-ray data on Cyg X-3 allow detailed modelling of the conditions in the inner disk and corona, the source of the jet energy. Study of the periodic nature of particle injection into the jet may yield an important clue to the nature of the particle injection mechanism. And the time scale of jet evolution is much shorter than for extragalactic sources, allowing the possibility of observing a large number of flares to determine general characteristics.

The radio emission was discoverd in 1971 (Braes and Miley 1972) and attracted wide attention in September, 1972 when the radio flux density rose from $\sim 10 \mathrm{mJy}$ to $\sim 20 \mathrm{Jy}$ over a span of a few days (Gregory et al. 1972). The spectral evolution of this flaring event led to the interpretation of the radio waves as synchrotron emission from an expanding plasma of relativistic electrons. Measurements (Geldzahler et al. 1983) several weeks after another giant flare show the source to have become slightly resolved in the north-south direction, demonstrating expansion and suggesting a collimated (jet) morphology.

We have carried out a program of measurements of the spectral evolution of Cyg X-3 at times of low flux density that has shown the radio emission may at all times be described as a series of overlapping flares (Molnar, Reid, and Grindlay 1984, 1985). Flare amplitudes range from tens of milliJanskys to tens of Janskys (the giant flares), the larger flares being less common. While the amplitudes vary from one flare to the next, the time from the beginning of one flare to the beginning of the next is fixed at $4.95 \mathrm{~h}$, some $3 \%$ longer than the orbital period. Each flare shows the spectral evolution expected of an adiabatically expanding synchrotron source: the flux density peaks sooner and at a greater value at higher frequencies (van der Laan 1966). We analyzed a typical flare under this hypothesis assuming the total energy in the flare was less than the energy emitted in the X-rays during the flare to predict a range of expansion velocities of $.05 c-.25 c$ (Molnar, Reid, and Grindlay 1984).

As a preliminary VLBI study we took 6 hours of data on each of 2 days, 5 and 8 February, 1985. We used a 5 element array at $1.3 \mathrm{~cm}$ wavelength recording with the MkIII recorders in Mode A for maximum bandwidth. Measurements of 
interstellar scattering at longer wavelengths implied that we would have to use the shortest wavelength possible to see a flare become larger than its scattering size before it was too faint to detect.

We fit all the data to a model in which the source is an elliptical Gaussian of fixed axial ratio, $A$, and orientation, with the FWHM major axis expanding at a constant rate, $v$, from zero size at flare onset, and convolved with a Gaussian scattering disk of FWHM $\theta_{\text {scat }}$. The orientation $\left(5^{\circ}\right)$ was fixed by previous measurements and the flare onset by the light curve. The probability of obtaining a higher $\chi^{2}$ than our minimum value was .20 ; the best fit parameters $\left(v, A, \theta_{\text {scat }}\right)$ were $(.40 \mathrm{mas} / \mathrm{hr}, 2.2, .68 \mathrm{mas})$ with asymmetric statistical errors less than $15 \%$ (1- $\sigma)$. Wilkinson et al. presented high accuracy scattering data at this meeting that predict a scattering size of $0.68 \pm .09 \mathrm{mas}$, in agreement with the value we derive, supporting the validity of our model.

We chose the model above because our data are insufficient to make independent hybrid maps at each time step. The data and the model both suffice to determine the features of greatest interest: significant deviations from circularity and significant changes in size. Our main conclusions are that with greater than $5-\sigma$ certainty, the source is elongated and is expanding along its major axis. For our best values of the externally fixed parameters (orientation and time of flare onset), we can also conclude that with greater than 5- $\sigma$ certainty the source is also expanding along its minor axis. Changing the externally fixed parameters within their allowed range, the first two conclusions remain unchanged but values can be found for which this third conclusion is not significant.

We have also fit a two-sided jet model that is mathematically similar to the model above and allows a direct comparison with our earlier prediction. It consists of two identical circular Gaussian sources that have a an apparent transverse motion from the center as well as an intrinsic expansion. For a distance of $10 \mathrm{kpc}$, the fit yields a transverse motion of .20c, similar to that of SS433, and an expansion velocity of $.13 c$, within our predicted range of velocities.

We are currently working on a more extensive data base that will be able to confirm and extend the conclusions reached here. Only the short baselines show significant correlated flux because of the large scattering size. As short baselines in the current network do not tend to be connected, these data do not have any reliable closure phase measurements. In this regard the first few stations of the VLBA (Pietown, Los Alamos, and Kitt Peak) will allow significantly improved measurements because they, used together with the VLA, Goldstone, and Owens Valley, offer a large number of interconnected short spacings.

Braes, L. L. E., and Miley, G. K. 1972, Nature, 237, 506.

Dickey, J. M. 1983, Ap. J. (Lett.), 273, L71.

Geldzahler, B. J., et al. 1981, Ap. J., 251, 230.

Gregory, P. C. 1972, Nature, 239, 439.

Molnar, L. A. 1985, Ph.D. thesis, Harvard University.

Molnar, L. A., Reid, M. J., and Grindlay, J. E. 1984, Nature, 310, 662.

Molnar, L. A., Reid, M. J., and Grindlay, J. E. 1985, Radio Stars, (R. M. Hjellming and D. M. Gibson, eds.), Astrophysics and Space Science Series, Reidel, Dordrecht, p. 329.

van der Laan, H. 1966, Nature, 211, 1131. 